\title{
The pathology of asbestos related disease
}

\author{
JMG DAVIS
}

A symposium on the pathology of asbestos related disease, held in Edinburgh on 27 and 28 April 1983, was attended by leading British research workers and hospital pathologists, and by doctors from the pneumoconiosis medical panels and the asbestos industry. The topics discussed were the relationship between lung dust burden and disease; the diagnosis of mesothelioma; the patterns of present day asbestosis; and the medicolegal requirements for determining when individual cases of pulmonary interstitial fibrosis, bronchial carcinoma, or mesothelioma are related to asbestos. Informal presentations were made by experts on the subject and this paper is a summary of the main conclusions drawn from these presentations and subsequent discussion.

\section{Relationship between lung dust burden and asbestos related disease}

The first attempts to estimate the asbestos content of lung tissue consisted of weighing the extracted dust residue or its mass estimation by $x$ ray diffraction. Such procedures were, however, suitable only for substantial amounts of asbestos and could not distinguish between fibres and rounded particles of the same mineral type. For this reason techniques requiring the counting of fibres were developed.

Most studies on lung asbestos content have used phase contrast light microscopy, and this technique is also used for most dust counts required in medicolegal cases because it is more readily available than electron microscopy. ${ }^{1-3}$ The phase contrast microscope cannot, however, resolve fibres less than $0.20 \mu \mathrm{m}$ in diameter, and scanning and transmission electron microscopy are increasingly used for research purposes to count the whole spectrum of fibres in lung tissue. ${ }^{4}$ Apart from advantages stemming from greater resolution, electron microscopes can be combined with systems for energy dispersive $x$ ray analysis that allow identification of individual mineral fibres.

Three main methods of recovering fibres from

Address for reprint requests: Dr JMG Davis, Institute of Occupational Medicine, Roxburgh Place, Edinburgh, EH8 9SU.

Accepted 10 August 1984 lung tissue have been used. These are tissue digestion, with the use of either potassium hydroxide ${ }^{1}$ or sodium hypochlorite,${ }^{5}$ and tissue ashing in nascent oxygen. ${ }^{6}$ There is some evidence that potassium hydroxide digestion is better for fixed tissue, while sodium hypochlorite produces the best results with fresh lung specimens. Ashing is very effective in destroying lung tissue but it has been suggested that this technique can result in the breakup of asbestos and the splitting of fibre bundles, so that fibre counts produced from ashed specimens tend to be higher than those where chemical digestion has been used.

For fibre counts using phase contrast microscopy, some workers have used counting chambers containing wet suspensions of dust while others collect the fibres on a membrane filter, which is cleared for microscopic examination. ${ }^{3}$ The latter technique has advantages in that a permanent preparation is produced that can be counted by several observers. In addition, the membrane counted samples allow superior resolution and counts can be five times greater than those obtained from the same sample viewed in a counting chamber. ${ }^{7}$ For electron microscopy fibres are collected on the same type of membrane filter but, while the scanning electron microscope can examine these filters in toto, after coating with gold or carbon, in the case of transmission electron microscopy carbon coating is followed by removal of the filter material with chloroform. Most laboratories counting asbestos fibres use the counting criteria recommended by the Asbestosis Research Council in $1971 .^{8}$ These criteria include the requirement that all fibres greater than $5 \mu \mathrm{m}$ in length and less than $3 \mu \mathrm{m}$ in diameter should be counted provided that they have an aspect ratio (length:diameter) of at least $3: 1$. No distinction is made between basically cylindrical fibres and elongated plates, and to some extent this is responsible for the large variety of different minerals found in lung tissue that are regarded as "fibrous." Some laboratories count fibres shorter than $5 \mu \mathrm{m}$, especially when using electron microscopy and these factors have to be considered when figures are compared. Dust counts obtained by transmission electron microscopy are usually at least an order of magnitude greater than the corresponding counts made by light microscopy. ${ }^{9}$ 
Size distributions obtained by electron microscopy for asbestos fibres recovered from lung tissue indicate that most fibres are either too short to be included in a count by optical microscopy or too fine to be resolved by this technique. ${ }^{9}$ For crocidolite, amosite, and chrysotile respectively the percentages of fibres shorter than $2.5 \mu \mathrm{m}$ have been reported as 56,34 , and 85 and the percentages of fibres less than $0.125 \mu \mathrm{m}$ in diameter as 67,16 , and $82 .{ }^{10}$ Almost none of the chrysotile fibres are large enough to be detected with an optical microscope, especially with the techniques needed to assess lung residue material.

Few interlaboratory comparisons of fibre counts for the same tissue specimens have been undertaken, but where this has occurred some counts have been several times higher than others. ${ }^{4}$ Differences of 2-3 fold can, however, occur between repeated counts on the same specimen in one laboratory. In lung tissue fibre concentration may be several times higher in samples collected only centimetres away from other samples. ${ }^{11}$ It has been suggested that some such differences, such as the lower concentration reported for the costophrenic angle might be due to ventilation patterns. Since dust counts are usually expressed in relation to the weight of dry lung tissue examined, different levels of fibrosis could be important. Badly fibrosed areas would produce a high dry weight and thus apparently low fibre counts. Apart from differences due to pathological reactions, several fold differences have been noted between the upper and lower lobes of the lung. Surprisingly, while asbestosis is usually more associated with the lower lobes than the upper, some workers have reported that the upper lobes have the highest dust counts. ${ }^{5}$

Fibre counts obtained from human lungs using phase contrast microscopy seldom exceed a magnitude of $10^{3}$ per gram of dried lung tissue, while with electron microscopy the corresponding figure is of the order of $10^{9} .{ }^{8}$ From published figures ${ }^{9-14}$ it would appear that the ranges of fibre counts associated with different disease states are as follows (for comparative purposes all figures have been reduced to the levels that would have been seen by phase contrast microscopy even if electron microscopy was used). Fibre counts in control cases without industrial exposure to asbestos range from 5000 to 100000 per gram of dried lung tissue. In cases of mesothelioma counts range from 20000 up to the maximum for asbestosis cases. Fibre counts in cases of mild asbestosis range from below 1000000 to 50000000 while of those with severe asbestosis have from 1000000 to several hundred million fibres per gram of dried lung tissue. The lung fibre counts in cases of bronchial carcinoma among asbes- tos workers correspond to the level of asbestosis present in the lung tissue. Fibre counts have also been undertaken for pleural plaques and these range from 500 to 15000 fibres per gram of dried plaque.

Studies of estimations of lung fibre content using transmission electron microscopy have produced interesting information relating fibre type and disease. While chrysotile fibres are present in most human lungs, there is little relationship between fibre number and grade of asbestosis. Similarly cases of mesothelioma in general have chrysotile counts no higher than those found in controls. ${ }^{12}$ The count of amphibole fibres present in lung tissue does, however, appear to be related to the severity of asbestosis and those with mesothelioma have far higher levels than controls. ${ }^{13}$ It would appear that chrysotile is either rapidly removed from lung tissue and never builds up to high levels or that only a small proportion of inhaled chrysotile is ever deposited in the alveoli. In cases where amphibole fibres have been known to form only a small proportion of total life time exposure, more amphibole than chrysotile is nevertheless found in the lungs at necropsy. This may occur in chrysotile miners, whose only amphibole exposure is to tremolite present in the chrysotile ore body in small amounts as a contaminant. ${ }^{14}$ There is evidence that pulmonary clearance of amphibole fibres over $20 \mu \mathrm{m}$ in length is virtually nil, and so the residue of long amphibole fibres in lung tissue may correspond closely to life exposure..$^{15}$ If this can be validated then lung dust counts of long amphibole fibres might be used to estimate exposure in past working conditions where no direct evidence is available. Animal experimentation has confirmed that, for a given dose, less chrysotile than amphibole is deposited and retained in lung tissue ${ }^{1617}$; and there may be little relationship between the magnitude and duration of chrysotile exposure and the final lung burden.

Apart from chrysotile and those amphibole minerals (chiefly crocidolite and amosite) considered as true asbestos, many other mineral fibres are found in human lung tissue at necropsy in numbers similar to asbestos fibres. In asbestos workers, however, the number of non-asbestos fibres is equally high in those with and without asbestos related disease. The following non-asbestos minerals can occur in fibrous form (aspect ratio $>3: 1$, diameter $<3 \mu \mathrm{m}$ ) and fibres have been recorded in human lung tissue ${ }^{18}$ :

$\begin{array}{lll}\text { Attapulgite } & \text { muscovite } & \text { vermiculite } \\ \text { mullite } & \text { orthoclase } & \text { talc } \\ \text { illite } & \text { rutile } & \text { glass fibre } \\ \text { pyroxene } & \text { silica } & \text { ferroactinolite } \\ \text { pyrophyllite } & \text { kaolin } & \text { gypsum } \\ \text { feldspar } & & \end{array}$

Of these, mullite is found most frequently and 
counts of mullite fibres may be very high, although most of the fibres are extremely short.

For many years pathologists without facilities for tissue digestion and dust extraction have relied on the presence of asbestos bodies to aid them in the diagnosis of asbestos related disease. Only relatively long fibres, however (and only a minority of these), ever become coated. ${ }^{3}$ Moreover, in both man and experimental animals "ferruginous" bodies are known to form round a variety of mineral fibres, so that the presence of an occasional body is not proof of asbestos exposure. In human lungs most bodies present have formed round amphibole fibres, ${ }^{19}$ but chrysotile asbestos bodies form frequently in some species of experimental animals ${ }^{20}$ and the relative absence of chrysotile associated bodies from human lungs is probably associated with the low long term retention of chrysotile in man. Cases of mesothelioma have been recorded where examination of several lung sections revealed no asbestos bodies yet lung fibre counts were as high as $\mathbf{3 7 5} 000$ per gram of dried tissue. It would appear therefore that actual lung fibre counts are essential for the correct diagnosis of doubtful lesions, particularly in medicolegal cases.

There is an urgent need for greater standardisation of the techniques used in different laboratories to study lung fibre content and pulmonary disease between laboratories. The use of potassium hydroxide digestion of lung tissue and the counting of fibres after collection on membrane filters may be the techniques of choice. An international counting study using a standard set of specimens would be particularly advantageous. Eventually all dust counts used in medicolegal cases should be undertaken by accredited laboratories, whose standards would be checked periodically by the circulation of control dust preparations. Phase contrast microscopy may be adequate for fibre counting where significant amphibole exposure has occurred, but for those exposed only to chrysotile electron microscopy is regarded as essential. Similarly, the recognition of mineral fibres that are not asbestos but could be implicated in disease can only be achieved by electron microscopy. In future electron microscope counts may be required for legal purposes so that all sizes of fibres are included, but much more work on the standardisation of such counts is required before this would be justified.

At present the importance of the low levels of chrysotile asbestos found in human lung tissue is unknown. Possibly with moderate exposures chrysotile reaches a balance in the lungs, where clearance equates with new deposition. ${ }^{21}$ Since this level is obviously relatively low it could be argued that chrysotile was responsible for pulmonary dis- ease only when exposure levels were very high and that modern factory levels are safe. It is, however, possible that a relatively short time in the lung is necessary for chrysotile fibres to cause disease and that subsequent removal from lung tissue is unimportant. Resolution of these problems is one of the most important outstanding areas of research on asbestos related disease. Another major problem is whether there is a threshold dose of asbestos fibre below which disease will not occur. Asbestosis and an increased incidence of bronchial carcinoma appear to occur only with moderately high dust levels in the lungs but many mesotheliomas have been found where lung dust counts fall within the range found in controls who have received no industrial exposure. It is important to determine whether these cases have been caused by asbestos and whether there is any risk to people whose only asbestos exposure comes from the normal urban environment.

\section{Diagnosis of mesothelioma}

For many years the existence of primary malignancies of the mesothelium was disputed by many authorities. It was not until Wagner et al in $1960^{22}$ described an association between pleural mesothelioma and crocidolite exposure in South Africa that the attention of pathologists was drawn to this tumour. It became apparent that the mesothelioma presented considerable diagnostic difficulties, partly because of its very variable histological appearance and partly because it was often difficult to distinguish from secondary serosal deposits of carcinoma. These difficulties led the UICC in 1965 to define diagnostic criteria for diffuse mesothelial tumours, and these have stood the test of time remarkably well. ${ }^{23}$ One of the UICC recommendations was that pathology reference panels should be established to coordinate mesothelioma diagnosis on both a national and an international level. In the United Kingdom a central Reference Panel of Experts became established to advise the already existing pneumoconiosis medical panels and to provide information for a mesothelioma register. The formal mesothelioma panel was subsequently discontinued and now each pneumoconiosis medical panel has its own advisers. These experts circulate cases of particular diagnostic difficulty to each other on an informal basis. It is thought that only $5-10 \%$ of the 500 mesotheliomas occurring annually in the United Kingdom present serious diagnostic difficulties at necropsy. Diagnosis during life presents more problems, however, and the pathological confirmation of mesotheliomas has in many cases to await necropsy. Some cases of 
mesothelioma are not identified clinically or radiologically during life. The degree of diagnostic uncertainty in difficult cases tends to be masked because the pneumoconiosis medical panels are constrained by the need for diagnosis on the balance of probability and this fact should be recognised by epidemiologists. Most pathologists find that pleural mesotheliomas are more common than the peritoneal variety, although occasional publications have reported the reverse. ${ }^{24}$

The histological diagnosis of biopsy material seems likely to remain the principal source of information during life, but the value of cytological and histochemical evidence from pleural effusions is worth emphasising. Cytopathology is considered by many to require considerable skill that may not always be available; some centralisation of expertise on a regional basis may be desirable. Nevertheless, recent advances in endoscopy, fine needle aspiration, and brush biopsy techniques have meant that most pathologists are becoming more familiar with judgements based on individual cells. Recently developed immunodiagnostic techniques may be important and some recommend that cells in suspect effusions should be routinely processed for transmission electron microscopy. Exfoliation does not necessarily occur, however, at all stages of a mesothelioma.

The accepted histopathological criteria for the classification and diagnosis of mesothelial tumours are based on the UICC recommendations of $1965 .^{23}$ Macroscopically, tumours can exist as solid masses, diffuse sheets, or multiple nodular forms, affecting the whole mesothelial surface and often with multiple adhesions and consolidation of the viscera. There is a tendency for pleural tumours to adopt the predominantly diffuse form and for peritoneal tumours to be predominantly of nodular type. The exclusion of a primary tumour in other organs by means of a careful necropsy is an important requirement in the diagnosis of mesothelioma. The gross appearance, however, is not pathognomonic for mesothelioma, and histological examination is essential.

The wide variations in microscopic appearance between different mesotheliomas, and often between different areas of the same tumour, may make histological identification difficult. Whenever possible tissue samples from different areas should be examined. Three histological varieties of mesothelioma are described: epithelial type - this tends architecturally to mimic an adenocarcinoma; connective tissue type - this tends architecturally to mimic a sarcoma; mixed type - this contains substantial elements of both epithelial and connective tissue forms.
The epithelial type of mesothelioma shows tubules, clefts, and papillary areas, usually with a single layer of cuboidal cells with relatively scanty cytoplasm and a vesicular nucleus, often with a single nucleolus. The cells lining the tubules and clefts have a "string of beads" appearance. If the cells are more columnar, have more cytoplasm, and have deeply staining nuclei, they are more likely to be of adenocarcinomatous than of mesotheliomatous origin. While papillary patterns are common in mesotheliomas, other areas may show more solid sheets, and in some cases there is difficulty in determining whether tumour cells are of epithelial or connective tissue origin.

The connective tissue element may adopt a frank fibrosarcomatous appearance with spindle shaped cells showing frequent mitoses. Areas of chondroid appearance, bone formation, and irregular patterns of collagen are occasionally found. In addition to the obviously malignant connective tissue areas, there are often zones of banal appearance, ranging from benign fibrosis to acellular hyaline material.

Metastases of mesothelioma do occur quite frequently despite previously published evidence to the contrary. The most frequent sites are lymph nodes and peribronchial lymphatic vessels. Less frequently spread to bone, adrenal glands, kidneys and other sites is observed. The histological appearance of metastases may be of epithelial, connective tissue, or mixed types.

The use of special stains may be helpful in histopathological diagnosis. Most mesotheliomas secrete hyaluronic acid but not mucin (as determined by the diastase-periodic acid Schiff (PAS) procedure). The demonstration of carcinoembryonic antigen by immunoperoxidase staining techniques identifies a tumour as a carcinoma and excludes mesothelioma, ${ }^{25}$ while keratin is found in mesotheliomas (as well as squamous carcinomas) but is not a feature of adenocarcinomas. Other frequently used stains are alcian blue, toluidine blue, colloidal iron, and azure A. Formol saline fixation has been found to be as good as the acetic acid fixation originally proposed for mesothelioma and is nowadays more commonly used.

Exfoliative cytology may be particularly helpful in the establishment of diagnosis during life. ${ }^{26} 27$ Fluids from pleural or peritoneal effusions frequently contain large numbers of cells and the fluid provides a sufficiently nutritive broth for the cells to remain viable for up to 48 hours. This makes the transportation of material to regional centres possible. Ideally a study of exfoliative material should be combined with conventional histopathological examination of a cell pellet, and where possible the pellet should be examined also by transmission electron microscopy. 
Special stains are not thought to be particularly helpful in aiding cytopathological diagnosis. If hyaluronic acid is present in the effusion, however, this is an indication of mesothelioma, while positive staining of cells with PAS and diastase effectively excludes this diagnosis. The degree of exfoliation depends on the stage of the tumour and on the histological type. Despite these problems the reliability of the cytopathological diagnosis in cases of mesothelioma has been encouraging, only $15 \%$ of eventually proved mesotheliomas having been wrongly diagnosed on the basis of exfoliative studies as "other" tumours. ${ }^{26} \mathrm{Up}$ to $25 \%$ of effusions from mesotheliomas subsequently confirmed at necropsy, however, showed only a lymphocytic pattern or were completely acellular.

Cytopathological discrimination between benign and malignant mesothelial cells may present some difficulties, but malignant cells are usually larger and more pleomorphic and have more irregularity of the nuclear chromatin pattern. Malignant mesothelial cells also show denser cytoplasmic staining around the nucleus, which causes apparent exaggeration of the soft "lacy" appearance of the peripheral cytoplasm. This is seen to a lesser extent in benign mesothelial cells and is due to extensive formation of microvilli. Irregularities of nuclear and nucleolar outline at light microscopy level are minimal when compared with those seen in exfoliated carcinoma cells.

Transmission electron microscopy of solid biopsy specimens or cell pellets derived from exfoliated cells may be useful in the diagnosis of mesothelioma despite the fact that there appear to be no "marker" organelles specific for mesothelioma cells. ${ }^{28} 29$ Features such as the presence of secretory vacuoles, marked aggregation of microfilaments with desmosomal involvement, microvilli with rootlet formation, and a prominent basement membrane are in general associated with carcinoma rather than mesothelioma cells. Features suggestive of mesothelioma include long, slender, curving microvilli, microluminal spaces, long desmosomes, intracellular lipid and glycogen, and bundles of perinuclear cytoplasmic filaments not associated with desmosomes. If these features are present in combination, the evidence for a mesothelial origin is particularly strong. An incomplete basement membrane, so that there is a close apposition between collagen fibrils and cell membrane, has so far been observed only with mesothelioma and not carcinoma. A highly irregular nuclear outline and chromatin staining pattern seen by either light microscopy or electron microscopy suggests a carcinoma, while a feature of mesothelial cells is that the rather smooth nuclear outline visible with the light microscope can be seen by TEM to consist of many small, sharp nuclear indentations.

\section{Patterns of asbestosis seen by pathologists today}

The classical pattern of advanced asbestosis is one of severe pulmonary interstitial fibrosis that produces irregular opacities on chest radiographs and is seen histologically as severe, diffuse interstitial fibrosis, which may produce destruction of the alveolar architecture and microcyst formation (honeycomb lung). In Britain this pattern is now rarely seen. Current patients are frequently survivors from earlier years of relatively high dust exposure, who have mild to moderate asbestosis but are dying of related malignancy or of non-pulmonary disease. Asbestosis is seldom sufficiently severe for it to be listed as the sole cause of death on death certificates. Only 29 such cases were recorded in the figures of the Office of Population Censuses and Surveys for 1979.

The current pattern of asbestosis is one of relatively mild fibrosis which progresses slowly, if at all. The gross appearance of the lungs at necropsy is not always useful and a histological diagnosis is helpful. Correct inflation of lungs and the use of the barium sulphate technique of Heard makes the demonstration of fibrosis easier. It is considered that the best area to pick up minimal change is the subpleural zone, where in the earliest stages evidence of fibrosis may extend inwards for only three or four alveoli. Useful sites for histological sampling are the lower part of the upper lobe and the posterior and diaphragmatic (near the costophrenic angle) aspects of the lower lobe. On histological examination early asbestosis appears as patchy fibrosis seen either as foci in the centrilobular position, often associated with carbon pigmentation, or subpleurally, where its distribution may be either linear or wedge shaped. Later a fine fibrosis of the alveolar wall may be seen. The lower lobe of the lung tends to be more extensively affected by fibrosis then the upper. In a clinical sense the mild degree of asbestosis now normally seen is of little importance. It is, however, critical in determining whether an associated carcinoma should be attributed to occupational exposure to asbestos. Pleural plaques occur frequently in patients with a history of asbestos exposure. While patterns of asbestosis appear to differ little throughout Britain, it is suggested that the incidence of asbestosis occurring with pleural and peritoneal mesotheliomas may vary. In most places peritoneal mesotheliomas appear most likely to be associated with asbestosis but exceptions do occur. ${ }^{24}$

Criteria used to grade the severity of asbestosis still vary between different pathologists and it seems important that one set of standards should be 
adopted, if possible on a worldwide basis. One suggestion for a grading system was published in $1973^{30}$ and a recent monograph on asbestos associated diseases publishedrin the United States made similar recommendations. ${ }^{31}$ Four grades of severity are proposed. Grade 1 is used for cases of fibrosis affecting the wall of at least one respiratory bronchiole with or without extension into the septum of the immediately adjacent layer of alveoli. No fibrosis is present in more distal alveoli. The appearances in grade 2 fibrosis are those of grade 1 but in addition alveolar ducts or two or more layers of adjacent alveoli are affected. There must still be a zone of non-fibrotic alveolar septum between adjacent bronchioles. In grade 3 fibrosis there is coalescence of fibrotic change such that all alveoli between at least two adjacent bronchioles have thickened fibrotic septa. Some alveoli may be obliterated completely. In grade 4 fibrosis there is formation of new spaces of a size larger than alveoli ranging up to as much as $1 \mathrm{~cm}$ (honeycombing). Spaces may or may not be lined by rounded epithelium.

The medicolegal problem: is disease related to asbestos exposure?

In Britain claims for industrial injuries benefit in relation to asbestosis and certain other industrial diseases are decided by the pneumoconiosis medical panels according to the statutory regulations. They are concerned only with prescribed industrial diseases, which are diseases considered to result from specific risks of occupation and not risks that affect the whole population. In each individual case the link with occupational risks must be recognisible. Industrial injuries benefit for prescribed industrial diseases is paid only to people who have been employed in prescribed occupations and in the case of the asbestos industry these are: $(a)$ any occupation requiring the working or handling of asbestos; $(b)$ the manufacture or repair of asbestos textiles or articles containing or composed of asbestos; (c) cleaning of any machinery or plant used in any of the foregoing operations and of any chambers, fixtures, and appliances for the collection of asbestos dust; (d) substantial exposure to dust arising from the foregoing occupations.

In practice, the eligibility of any claimant for compensation is decided by an insurance officer before the individual is examined by doctors from the pneumoconiosis medical panels, who decide only on the type and extent of the disease and assess disability. The panel doctors are, however, greatly assisted by information provided by chest physicians and pathologists. For the purpose of compensation, diagnosis is made on the balance of probability rather than that the prescribed disease cannot be excluded. In Britain once the diagnosis of pneumoconiosis or mesothelioma has been made by the panel doctors itcannot be changed as a result of subsequent clinical investigation and the patient is always "deemed" to have the condition diagnosed. This still applies after death. If a person dies from a condition incorrectly diagnosed as pneumoconiosis or mesothelioma during life he is still "deemed" to have died of this condition and the case is recorded as such in official statistics.

Difficulties frequently arise, particularly in cases of pulmonary fibrosis presenting a similar picture to that of asbestosis. A case of cryptogenic fibrosing alveolitis with substantial exposure to asbestos would probably be recorded as asbestosis and compensated accordingly. Someone with mesothelioma, which is a prescribed disease in its own right, is compensated automatically if there is any evidence of occupational asbestos exposure, but bronchial carcinoma in asbestos workers is considered to be caused by asbestos only if asbestos is also present. It was recently proposed that the legislation should be amended so that the occurrence of pleural plaques or diffuse pleural thickening would also be considered as evidence of asbestos exposure sufficiently severe to be the causative agent in cases of bronchial carcinoma. ${ }^{32}$ Reports show, however, that cases from the general population with plaques fail to show a greater history of asbestos exposure than those without ${ }^{33}$ and that dockyard workers exposed to asbestos who develop plaques do not show any significant increase in incidence of bronchial carcinoma compared with those who do not (Rossiter CE, Coles RM, Jackaman I. Proceedings of Sixth International Conference on Pneumoconiosis. Bochum, 1983, to be published).

Because of the medicolegal implications, the correct diagnosis of early asbestosis remains one of the greatest problems for pathologists. Focal deposits of fibrous tissue around the terminal and respiratory bronchioles may be caused by, many types of dust and it has been reported that as many as one third of all those with bronchial carcinoma have focal pulmonary fibrosis due to tobacco smoke. There are also considerable difficulties in distinguishing more advanced cases of asbestosis from cryptogenic fibrosing alveolitis, particularly in those who may have had some asbestos exposure. It is suggested, however, that the absence of pleural adhesions or fibrosis, the presence of honeycomb cysts with smooth muscle hyperplasia, and lymphoid hyperplasia all favour a diagnosis of cryptogenic fibrosing alveolitis.

Since the lesions of asbestosis are not specific in their own right, evidence of the presence of asbestos 
fibres within the lung tissue is essential in establishing a diagnosis. In the past the recognition of asbestos bodies in histological sections has been widely used for this purpose and is still useful where large numbers of these structures are present. Many cases occur, however, where no asbestos bodies are found in several lung sections are and yet fibre counts obtained after tissue digestion have been high. More emphasis is now being placed on fibre counts and for this reason standardisation of extraction and counting procedures is important. Since some asbestos fibres are present in all lungs a fibre level at which asbestos exposure is considered to have caused disease must be established. On this subject opinions differ. Some pathologists suggest that for phase contrast microscopy 1000000 fibres per gram of lung tissue represent a threshold below which fibrosis is unlikely to occur. Others believe that a figure of 250000 fibres per gram should be accepted. ${ }^{34}$ Those exposed only to chrysotile present a problem in that this material can seldom be seen in tissues with a light microscope. In these cases the use of electron microscopy, which also allows fibre identification, may be particularly helpful.

There is a strong feeling that the present custom of attributing pulmonary disease to asbestos exposure on an "all or nothing" basis is unsatisfactory. Many pathologists suggest that it would be better to attempt to apportion causality in many cases. For example, this might be desirable where bronchial carcinoma and mild asbestosis are found in the lungs of a heavy smoker, and where pulmonary fibrosis has presented as cryptogenic fibrosing alveolitis in life and shown the histological pattern of this disease at necropsy but where there is evidence of asbestos exposure.

The following were speakers, chairmen, or rapporteurs at the meeting and all have reviewed the contents of this paper: JMG Davis (convener), T Ashcroft, RE Bolton, B Butler, B Corrin, JE Craighead, AT Edwards, PC Elmes, J Enticknop, JC Gilson, AD Jones, AW Jones, JSP Jones, D Lamb, A Morgan, TJG Phillips, F Pooley, VA Ruckley, R Seal, A Seaton, JC Wagner, F Whitwell.

\section{References}

' Ashcroft T, Heppleston AG. The optical and electron microscopic determination of pulmonary asbestos fibre concentration and its relation to the human pathological reaction. J Clin Pathol 1973;26:224-34.

${ }^{2}$ Whitwell F, Scott J, Grimshaw M. Relationship between occupations and asbestos fibre content of the lungs in patients with pleural mesothelioma lung cancer and other diseases. Thorax 1977;32:377-86.

${ }^{3}$ Morgan A, Holmes A. Concentrations and dimensions of coated and uncoated asbestos fibres in the human lung. Br J Ind Med 1980;37:25-32.

${ }^{4}$ Pooley FD, Clark NJ. Quantitative assessment of inorganic fibrous particulates in dust samples with an analytical transmission electron microscope. Ann Occup Hyg 1979;22:253-72.

${ }^{s}$ Sebastien P, Fondimare A, Bignon J, Monchaux G, Desbordes J, Bonnaud G. Topographic distribution in relation to occupational and non-occupational exposure. In Walton WH, ed. Inhaled particles IV. Oxford: Pergamon Press, 1977:435-46.

${ }^{6}$ Le Bouffant L, Bruyere S, Martin JC, Tichoux G, Normand C. D'Amiante et les formations minérales diversus recontrées dans les poumons asbestosiques. $\mathrm{Rev} \mathrm{Fr}$ Mal Respir 1976;4,suppl 2:121-40.

${ }^{7}$ Morgan A, Holmes A. Concentrations and characteristics of amphibole fibres in the lungs of workers exposed to crocidolite in the British gas mask factories and elsewhere, during the second world war. Br J Ind Med 1982;39:62-9.

${ }^{8}$ Asbestosis Research Council. The measurement of airborne asbestos dust by the membrane filter method. Rochdale: Asbestos Research Council, 1971. (Technical Note 1.)

${ }^{9}$ Pooley FD, Clark NJ. A comparison of fibre dimensions in chrysotile, crocidolite and amosite particles from samples of airborne dust and from post-mortem lung tissue specimens. In: Wagner JC, ed. Biological effects of mineral fibres. Lyon: International Agency for Research on Cancer, 1980:79-87. (IARC Scientific Publication 30.)

${ }^{10}$ Pooley FD. Tissue burden studies. In: National Board of Occupational Safety and Health Research Department, Sweden. Short and thin mineral fibres: identification, exposure and health effects. Proceedings of symposium. Solna: National Board of Occupational Safety and Health, 1983:95-125.

"Morgan A, Holmes A. Distribution and characteristics of amphibole asbestos fibres, measured with the light microscope, in the left lung of an insulation worker. $\mathrm{Br}$ $J$ Ind Med 1983;40:45-50.

12 Wagner JC, Berry G, Pooley FD. Mesotheliomas and asbestos type in asbestos textile workers: a study of lung contents. $\mathrm{Br}$ Med J 1982;285:603-6.

${ }^{13}$ Wagner JC, Pooley FD, Berry G, et al. A pathological and mineralogical study of asbestos related deaths in the United Kingdom in 1977. Ann Occup Hyg 1982; 26: 423-30.

${ }^{14}$ Pooley FD. An examination of the fibrous mineral content of asbestos lung tissue from the Canadian chrysotile mining industry. Environ Res 1976; 12: $281-98$.

15 Timbrell V. Deposition and retention of fibres in the human lung. Ann Occup Hyg 1982;26:347-68.

${ }^{16}$ Wagner JC, Berry G, Skidmore JW, Timbrell V. The effects of the inhalation of asbestos in rats. $B r J$ Cancer 1974;29:252-69.

${ }^{17}$ Davis JMG, Beckett S1, ?olton RE, Collings P, Middleton AP. Mass and number of fibres in the pathogenesis of asbestos related lung disease in rats. $\mathrm{Br} J$ Cancer $1978 ; 37: 673-88$.

${ }^{18}$ Churg A. Nonasbestos pulmonary minerals fibres in the general population. Environ Res 1983;31:189-200.

${ }^{19}$ Churg A, Warnock ML. Analysis of the cores of asbestos bodies from members of the general population: patients with probable low-degree exposure to asbes- 
tos. Am Rev Respir Dis 1979;120:781-6.

${ }^{20}$ Davis JMG. Further observations on the ultrastructure and chemistry of formation of asbestos bodies. Exp Mol Pathol 1970;13:346-58.

${ }^{21}$ Bolton RE. Vincent JH, Jones AD, Addison J, Beckett ST. An overload hypothesis for pulmonary clearance of UICC amosite fibres inhaled by rats. $\mathrm{Br} \mathrm{J}$ Ind $\mathrm{Med}$ 1983;40:264-72.

${ }^{22}$ Wagner JF, Sleggs CA, Marchand P. Diffuse pleural mesotheliomata and asbestos exposure in the north western Cape Province. Br J Ind Med 1960;17:26071.

${ }^{23}$ Working Group on Asbestos and Cancer. Report and recommendations. Ann NY Acad Sci 1965;132:70621.

${ }^{24}$ Browne K, Smither WJ. Asbestos-related mesothelioma: factors discriminating between pleural and peritoneal sites. Br J Ind Med 1983;40:145-52.

${ }^{25}$ Whitaker D, Shilkin KB. Carcinoembryonic antigen in tissue diagnosis of malignant mesothelioma. Lancet 1981;i: 1369.

${ }^{26}$ Butler EB, Berry AV. Diffuse mesotheliomas: diagnostic criteria using exfoliative cytology. In: Bogovski $\mathbf{P}$, ed. Biological effects of asbestos. Lyon: International Agency for Research on Cancer 1973:68-73. (IARC Scientific Publication 8.)

${ }^{27}$ Butler EB, Johnson NF. The use of electron microscopy in the diagnosis of diffuse mesothelioma using human pleural effusions. In: Wagner JC, ed. Biological effects of mineral fibres. Lyon: International Agency for Research on Cancer 1980:409-18. (IARC Scientific Publication 30.)

${ }^{28}$ Wang NS. Electron microscopy in the diagnosis of pleural mesotheliomas. Cancer 1973;31:1046-54.

${ }^{29}$ Stoebner P, Brambilla E. Ultrastructural diagnosis of pleural tumours. Pathol Res Pract 1982;173:402-16.

${ }^{30}$ Hinson KF. Otto H, Webster I, Rossiter CE. Criteria for the diagnosis and grading of asbestosis. In: Bogovski $\mathrm{P}$, ed. Biological effects of asbestos. Lyon: International Agency for Research on Cancer, 1973:54-8. (IARC Scientific Publication 8.)

${ }^{31}$ Craighead JE. Report of the Pneumoconiosis Committee of the College of American pathologists and the National Institute for Occupational Safety and Health Arch Pathol Lab Med 1982;106:543-97.

32 Davies D. Asbestos related disease without asbestosis Br Med J 1983;287:164.

${ }^{33}$ British Thoracic and Tuberculosis Association and Medical Research Council Pneumoconiosis Unit. A survey of pleural thickening: Its relation to asbestos exposure and previous pleural disease. Environ Res 1972;5:142-51.

${ }^{34}$ Seal RME. Current views on pathological aspects of asbestosis. In: Wagner JC, ed. Biological effects of mineral fibres. Lyon: International Agency for Research on Cancer, 1980:217-37. (IARC Scientific Publication 30.) 